\title{
Coverage, Probability of SNR Gain, and DOR Analysis of RIS-Aided Communication Systems
}

\author{
Liang Yang, Yin Yang, Mazen O. Hasna, Senior Member, IEEE and Mohamed-Slim Alouini, Fellow, IEEE
}

\begin{abstract}
Recent studies have shown that reconfigurable intelligent surfaces (RISs) can help in extending the wireless coverage. However, no work has quantitatively analyzed the coverage. In this letter, we present the coverage analysis for an RIS-aided communication system. For comparison, the coverage analysis for relaying systems and direct-link transmission systems are also derived. Furthermore, we provide the analysis for the probability of signal-to-noise ratio (SNR) gain of the RIS over the direct link transmission. Finally, we analyze the delay outage rate of the RIS in achieving ultra-reliable low-latency transmissions. The results show that using the RIS can expand the coverage, increase the SNR gain, and reduce latency of the links.
\end{abstract}

Index Terms-Coverage distance, delay outage rate, probability of SNR gain (PSG), relay, reconfigurable intelligent surface (RIS).

\section{INTRODUCTION}

As one of the key technologies for future wireless networks, reconfigurable intelligent surfaces (RISs) have been widely used in various fields. The RISs do not need any power supply, complicated signal processing, or encoding and decoding processes, which are especially used to improve the signal quality at the receiver [1][2]. Indeed, the RISs are unique in that they enable telecom operators with controllable environment to realize the distribution of the whole network by modeling the environment object and fully controlling the electromagnetic response [3]. Notice that the RIS is also known as an intelligent reflecting surface (IRS) and the authors in [4]-[6] proposed using the IRSs to realize high spectrum and energy efficiency in the wireless communication. In particular, the authors in [4] emphasized the main differences and the

Copyright (c) 2015 IEEE. Personal use of this material is permitted. However, permission to use this material for any other purposes must be obtained from the IEEE by sending a request to pubs-permissions@ieee.org

Manuscript received Mar 15, 2020; revised Apr 9, 2020; accepted Apr 10, 2020. This work was in part supported by the National Natural Science Foundation of China (NSFC) under Grant 61671160, the Department of Education of Guangdong Province (No. 2016KZDXM050), the Hunan Natural Science Foundation under Grant (No.2019JJ40043), the Science and Technology Program of Guangzhou (No.201904010249), and the Science and Technology Program of Changsha (kq1907112). The review of this paper was coordinated by Dr. Hien Ngo. (Corresponding author: Liang Yang)

L. Yang is with the College of Computer Science and Electronic Engineering, Hunan University, Changsha 410082, China, and also with the State Key Laboratory of Integrated Services Networks, Xidian University, Xian 710126, China.(e-mail: liangy@hnu.edu.cn).

Y. Yang is with the College of Computer Science and Electronic Engineering, Hunan University, Changsha 410082, China.(e-mail: yy19971417@163.com).

M. O. Hasna is with the Department of Electrical Engineering, Qatar University, Doha 2713, Qatar (e-mail: hasna@qu.edu.qa).

M.-S. Alouini is with the Computer, Electrical, and Mathematical Science and Engineering Division, King Abdullah University of Science and Technology, Thuwal 23955, Saudi Arabia (e-mail: slim.alouini@kaust.edu.sa). competitive advantages of the IRS through comparison with other related technologies. RISs and their typical applications in wireless communication systems have been extensively studied in the literature. For instance, the authors in [7] studied applying the RIS to the downlink multi-user communication. In addition, a structure with a holographic multipleinput multiple-output surface (HMIMOS) was proposed in [8]. However, most results are only applicable to the ideal phase shift models. Therefore, the authors in [6][9] studied the performance loss due to discrete or practical phase shifts.

On the one hand, as mentioned in many references, using RISs can improve the coverage of wireless communication systems, which is a very intuitive conclusion. So far, regarding the performance analysis works, most of the results are available for the common performance indicators, such as average capacity, bit error rate, and outage probability. However, only few works have presented the quantitative coverage analysis for the conventional relaying systems. In particular, in [10], the authors analyzed the coverage of a decode-and-forward (DF) millimeter wave (mmwave) relaying system and compared it with the direct-link transmission case. The results in [10] showed that using relays can significantly improve the coverage performance. In [11], the authors considered a two-way relaying network with spectrum sharing and multiuser diversity, and analyzed the coverage by starting from the outage probability computation. However, the coverage analysis for the RIS-aided wireless system is still not reported in the literature, which is one of the main motivations of this work.

Moreover, compared with the direct link transmission, there is a great interest in how much signal-to-noise ratio (SNR) gain the relay system can provide. For a single-input singleoutput (SISO) system, the probability of SNR gain (PSG) was presented in [12]. The results in [12] indicated that the PSG mainly depends on the ratio of average SNRs. In [13], the authors studied the PSG with the multiple-input multipleoutput (MIMO) relaying systems with the Alamouti code scheme, and their results show that MIMO relay systems can obtain the SNR gain even if the diversity order is smaller than that of the single-hop MIMO systems. To the best of the authors' knowledge, the analysis for the PSG of RISaided systems over direct link transmission systems is still not reported in the literature, which is another motivation of this work.

Finally, achieving ultra-high reliability and low latency communication (URLLC) is a major requirement in the 5th generation $(5 \mathrm{G})$ communication systems. The use of dataoriented performance indicators to analyze the transmission 


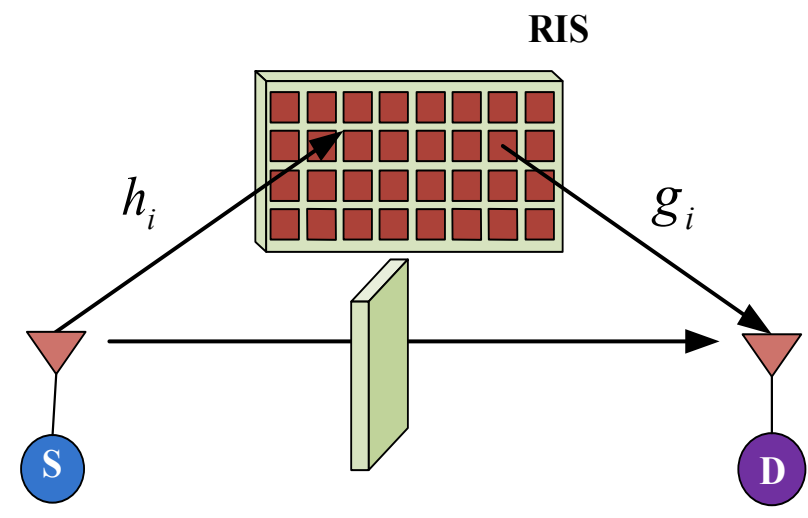

Fig. 1. An RIS-aided wireless communication system model.

performance of small data in fading channels has been studied in many literatures. For instance, in [14], the authors proposed a data-oriented method that uses the principle of optimizing the transmission strategy of a single data session to improve the performance of the entire system and effectively meet the reliability and latency requirements of mission-critical applications. In [15], the authors analyzed the delay outage rate (DOR) for the ideal continuous rate and actual discrete rate adaptation schemes, and the results show that the discrete rate adaptation with adaptive modulation and coding (AMC) is not as good as the expected optimal rate adaptation (ORA). However, the DOR analysis for the RIS-aided wireless communication transmission has not been reported in the literature, which is the last motivation of this work.

In this letter, for the RIS-aided communication systems, we present the analysis for the coverage, the PSG, and the DOR. For comparison, we also analyze the coverage and the DOR of dual-hop relaying systems. Furthermore, we investigate the impact of relative link gains (RLGs) on the SNR gain. Finally, the results show that the use of the RIS can provide larger coverage, increase the SNR gain, and reduce the DOR.

\section{SySTEM AND CHANNEL MODELS}

As shown in Fig. 1, consider an RIS-aided communication system, which includes a source (S), a destination (D) and an RIS that is composed of $N$ reflecting meta-surfaces, where $\mathrm{S}$ and $\mathrm{D}$ are equipped with only one antenna. We assume that the RIS is placed between $\mathrm{S}$ and $\mathrm{D}$, and direct-link transmission does not exist due to the blockage, then an intelligent environment is created by the RIS to improve the signal quality. Furthermore, we assume that the RIS can obtain the full channel state information (CSI) of the channels to realize the phase shift, thereby maximizing the received SNR at D. Finally, similar to the assumption in [5], we assume that only the signal reflected by the RIS first time is considered, and those reflected by the RIS two or more times are ignored.

From Fig. 1, we can see that the RIS is working like a relay. However, their working principles are completely different and the detailed difference between them has been presented in [16]. More specifically, relays are used to amplify or decode and forward signals and extend the coverage of a wireless network, while the spectrum efficiency loss happens due to the use of multiple time slots. However, the RISs just passively reflect signals without additional power to optimize the phases of the reflection and maximize the SNR, which eventually improves the end-to-end quality of the communication link.

We assume that the channel is a quasi-static flat Rayleigh fading channel without interference. Therefore, the received signal at $\mathrm{D}$ can be expressed as

$$
y=\sqrt{P_{s}}\left[\sum_{i=1}^{N} h_{i} \varrho_{i} g_{i}\right] x+n_{0},
$$

where $P_{s}$ is the transmit power at $\mathrm{S}, x$ is the transmit signal with unit energy, and $n_{0}$ is the additive white Gaussian noise (AWGN) with the mean of zero and the variance of $N_{0}$. In (1), $\varrho_{i}=\varpi_{i}\left(\phi_{i}\right) e^{j \phi_{i}}$ is the reflection coefficient produced by the $i$ th reflector of the RIS, where $\varpi_{i}\left(\phi_{i}\right)=1$ for the ideal phase shifts $(i=1,2, \ldots, N)$. In addition, $h_{i}$ and $g_{i}$ are the channel gains with $h_{i}=d_{1}^{-v / 2} \alpha_{i} e^{-j \theta_{i}}$ and $g_{i}=d_{2}^{-v / 2} \beta_{i} e^{-j \varphi_{i}}$, where $d_{1}$ and $d_{2}$ are the distances for the S-RIS link and RIS-D link, respectively, $v$ is the path-loss index, $\theta_{i}$ and $\varphi_{i}$ are the phases of the channel gains, and $\alpha_{i}$ and $\beta_{i}$ following a Rayleigh distribution are the amplitudes of the channel gains. Moreover, we assume that the RIS has the full knowledge of the channels phases of $h_{i}$ and $g_{i}$.

From [17], the path-loss model in RIS-aided communication systems has two cases, namely, near field and far field. In particular, when the distance between the source/terminal and the middle of the RIS is less than $\frac{2 D^{2}}{\lambda}$, where $D$ represents the maximum size of the RIS and $\lambda$ is the wavelength, the system model is classified as the near field case. Otherwise, the system model belongs to the far-field case. In this work, we consider the far field case. Then, the received SNR at D can be expressed as

$$
\gamma=\frac{\left|\sum_{i=1}^{N} \alpha_{i} \beta_{i} e^{j\left(\phi_{i}-\theta_{i}-\varphi_{i}\right)}\right|^{2} P_{s}}{N_{0} d_{1}^{v} d_{2}^{v}},
$$

In (2), to obtain the maximum value of $\gamma$, we need to eliminate the channel phases. Similar to the method in [5], by setting the phases $\phi_{i}=\theta_{i}+\varphi_{i}$ for $i=1,2, \ldots, N$, the maximal $\gamma$ can be written as [5]

$$
\gamma=\frac{\left(\sum_{i=1}^{N} \alpha_{i} \beta_{i}\right)^{2} P_{S}}{N_{0} d_{1}^{v} d_{2}^{v}}=\frac{A^{2} \bar{\gamma}}{d_{1}^{v} d_{2}^{v}},
$$

where $\bar{\gamma}=P_{s} / N_{0}$ denotes the average SNR and $A=\sum_{i=1}^{N} \alpha_{i} \beta_{i}$.

\section{Coverage Analysis}

In this section, our intention is to derive the outage probability expressions and then use them to analyze the coverage.

\section{A. Outage Probability Analysis}

Outage probability is defined as the probability that the effective received SNR $\gamma$ at D is less than a given value $\gamma_{\text {th }}$. Thus, we readily have $P_{\text {out }}=\operatorname{Pr}\left(\gamma<\gamma_{\text {th }}\right)$. 
For large number of reflecting meta-surfaces, i.e., $N \gg$ 1 , and according to the central limit theorem (CLT), $A$ is a Gaussian distributed random variable with the mean of $\frac{N \pi}{4}$ and the variance of $N\left(1-\frac{\pi^{2}}{16}\right)$ [1]. As a result, $A^{2}$ is a noncentral chi-square random variable with one degree of freedom and its probability density function (PDF) is given by [18]

$$
f_{A^{2}}(s)=\frac{1}{2 \sigma^{2}}\left(\frac{s}{\lambda}\right)^{-\frac{1}{4}} \exp \left(-\frac{s+\lambda}{2 \sigma^{2}}\right) I_{-\frac{1}{2}}\left(\frac{\sqrt{s \lambda}}{\sigma^{2}}\right) .
$$

where $I_{n}(z)$ is the modified Bessel function of the first class with order $n, \lambda=\left(\frac{N \pi}{4}\right)^{2}$, and $\sigma^{2}=N\left(1-\frac{\pi^{2}}{16}\right)$. According to (3), the PDF of $\gamma$ can be written as

$$
f_{\gamma}(\gamma)=\frac{1}{2 \sigma^{2} \bar{\gamma}}\left(\frac{\gamma}{\bar{\gamma} \lambda}\right)^{-\frac{1}{4}} \exp \left(-\frac{\gamma+\lambda \bar{\gamma}}{2 \bar{\gamma} \sigma^{2}}\right) I_{-\frac{1}{2}}\left(\frac{\sqrt{\gamma \lambda}}{\sqrt{\bar{\gamma}} \sigma^{2}}\right) .
$$

With (5), $P_{\text {out }}$ can be expressed as

$$
P_{\text {out }}=1-Q_{\frac{1}{2}}\left(\frac{\sqrt{\lambda}}{\sigma}, \frac{\sqrt{\gamma_{\mathrm{th}}}}{\sqrt{\bar{\gamma}} \sigma}\right),
$$

where $Q_{m}(a, b)$ is the Marcum Q-function defined in [19].

The above analytical result involves the Marcum Q-function, which can not provide any explicit insights. Also, it is difficult to use it to analyze the coverage and compare with other schemes. Thus, an asymptotic outage analysis is necessary. By assuming $d_{1}=d_{2}=\frac{d^{\mathrm{RPS}}}{2}$, from [20], the Marcum Q-function $Q_{m}(x, y)$ can be asymptotically expressed as

$$
Q_{m}(x, y) \leq(1-2 \varepsilon)^{-m} \exp \left(-\varepsilon y^{2}\right) \exp \left(\frac{m \varepsilon x^{2}}{1-2 \varepsilon}\right),
$$

where $\varepsilon$ is the Chernoff parameter $\left(0<\varepsilon<\frac{1}{2}\right)$. Since $\exp \left(\frac{1 / 2 \varepsilon x^{2}}{1-2 \varepsilon}\right)$ has a minimum value of $1, P_{\text {out }}$ can be asymptotically written as

$$
P_{\text {out }} \approx 1-(1-2 \varepsilon)^{-1 / 2} \exp \left(-b^{2} \varepsilon\right),
$$

where $b^{2}=\frac{\left(d^{R I S}\right)^{2 v} \gamma_{\mathrm{th}}}{4^{v-2} \bar{\gamma} N\left(16-\pi^{2}\right)}$. Above expression explicitly indicates that increasing $N$ can improve the system performance.

\section{B. Coverage Analysis}

In this subsection, we provide the coverage analysis to show the advantage of the RISs. For comparison, we also analyze the coverage for the relaying system. For fair comparison, we assume that the channel fading is the Rayleigh fading for all cases and all the links have the same average SNRs $\bar{\gamma}$.

According to [21], the cell coverage is related to the outage probability and is defined as the maximum distance at which the link quality at the cell edge is no less than a given SNR value $\gamma_{\mathrm{th}}$, with the probability $1-P_{\text {out }}$. Therefore, from (8), the coverage distance $d^{\mathrm{RIS}}$ can be approximated as

$$
d^{\mathrm{RIS}} \approx\left(\frac{N 4^{v-2} \bar{\gamma}\left(16-\pi^{2}\right) \ln \frac{(1-2 \varepsilon)^{-1 / 2}}{1-P_{\text {out }}}}{\gamma_{\mathrm{th}} \varepsilon}\right)^{1 /(2 v)} .
$$

For the direct-link transmission system, the link gain $|h|^{2}$ obeys the exponential distribution with parameter $\left(d^{\mathrm{D}}\right)^{v}$. Therefore, the distance $d^{\mathrm{D}}$ can be exactly expressed as

$$
d^{\mathrm{D}}=\left(\frac{\bar{\gamma} \ln \frac{1}{1-P_{\text {out }}}}{\gamma_{\text {th }}}\right)^{1 / v} .
$$

Notice that (10) can also be obtained from [21].

For the relaying system with the DF protocol, the end-toend outage probability is given by $P_{\text {out }}=\operatorname{Pr}\left\{\min \left(\gamma_{1}, \gamma_{2}\right)<\right.$ $\left.\gamma_{\text {th }}\right\}=1-\operatorname{Pr}\left\{\gamma_{1}>\gamma_{\text {th }}, \gamma_{2}>\gamma_{\text {th }}\right\}$, where $\gamma_{1}$ and $\gamma_{2}$ are the effective SNRs of the two hops. Without loss of generality, we assume that the relay is placed at the middle of the S-relay (R)-D link. Therefore, the distance $d^{\mathrm{R}}$ for the relaying system can be written as

$$
d^{\mathrm{R}}=\left(\frac{2^{v-1} \bar{\gamma} \ln \frac{1}{1-P_{\text {out }}}}{\gamma_{\text {th }}}\right)^{1 / v} .
$$

For tractable analysis, $v$ is set to 2. From (10) and (11), we have $d^{\mathrm{R}}=\sqrt{2} d^{\mathrm{D}}$, which means that the relaying system can obtain a higher coverage. Furthermore, from (9), (1 $2 \varepsilon)^{-1 / 2}>1$. Therefore, for sufficiently large $N$, we have $\frac{d^{\mathrm{RIS}}}{d^{\mathrm{R}}}>\left(\frac{N\left(16-\pi^{2}\right) \gamma_{\mathrm{th}}}{2 \bar{\gamma} \ln \frac{1}{1-P_{\text {out }}}}\right)^{1 / 4}>1$. Thus, it is clearly observed that $d^{\mathrm{RIS}}$ is always larger than $d^{\mathrm{R}}$ and $d^{\mathrm{D}}$, which indicates that using RISs can increase the received SNR and make the wireless communication systems have a larger coverage.

\section{Probability of SNR Gain}

In this work, with the coverage analysis, we also provide the analysis for the PSG.

According to [12], the PSG is defined as the ratio of the instantaneous SNRs. The authors in [12] considered the PSG by relays over the single-hop system. Thus, the PSG by RISs or relays over direct-link transmission systems can be expressed as

$$
\begin{aligned}
P_{c}^{\delta} & =\operatorname{Pr}\left\{\frac{\gamma_{\delta}}{\gamma_{\mathrm{D}}}>\eta\right\} \\
& =1-\int_{0}^{\infty} \operatorname{Pr}\left\{\gamma_{\delta}<\eta \gamma_{\mathrm{D}} \mid \gamma_{\mathrm{D}}=\gamma\right\} f_{\gamma_{\mathrm{D}}}(\gamma) d \gamma \\
& =1-\int_{0}^{\infty} F_{\gamma_{\delta}}(\eta \gamma) f_{\gamma_{\mathrm{D}}}(\gamma) d \gamma .
\end{aligned}
$$

where $\eta$ is the threshold value, $\delta \in\{\mathrm{R}, \mathrm{RIS}\}, \gamma_{\mathrm{R}}=$ $\frac{\bar{\gamma}}{\left(\frac{d^{\mathrm{R}}}{2}\right)^{v}} \min \left\{\left|h_{1}\right|^{2},\left|h_{2}\right|^{2}\right\}=G_{1} \min \left\{\left|h_{1}\right|^{2},\left|h_{2}\right|^{2}\right\}$ represents the instantaneous end-to-end SNR for the relaying system, $\gamma_{\mathrm{RIS}}=\frac{\bar{\gamma}}{\left(\frac{d \mathrm{RIS}}{2}\right)^{2 v}} A^{2}=G_{2} A^{2}$ denotes the instantaneous SNR for the RIS-aided system, $F_{X}(x)$ and $f_{X}(x)$ are the cumulative distribution function (CDF) and the PDF of the random variable $X$, and $\gamma_{\mathrm{D}}=\frac{\bar{\gamma}|h|^{2}}{\left(d^{\mathrm{D}}\right)^{v}}=G_{0}|h|^{2}$ represents the received SNR for the direct link transmission case.

Let $L_{1}=G_{1} / G_{0}$ and $L_{2}=G_{2} / G_{0}$ be called the RLGs that represent the proportions of average received SNRs.

For the Rayleigh fading channels, the PSG for the relaying system is given by [12, eq.(10)]

$$
P_{c}^{\mathrm{R}}(\eta)=\frac{L_{1}}{2 \eta+L_{1}} .
$$


From [22] and [23, eq.(13)], the PSG for the RIS-aided system can be readily evaluated as

$$
P_{c}^{\mathrm{RIS}}(\eta)=1-\left(\frac{\eta}{\eta+2 L_{2} \sigma^{2}}\right)^{\frac{1}{2}} e^{-\frac{\lambda L_{2}}{\eta+2 L_{2} \sigma^{2}}},
$$

Since we assume that both the relay and the RIS are placed in the middle positions of the S-D link, we have $L_{1}=L_{2}$, which makes the PSG reach its maximum value. In this case, as the communication environment becomes more decentralized, RLG increases with the increase of the path loss index.

\section{Delay outage rate}

In this section, we analyze the DOR performance of small data transmission in the flat Rayleigh fading channels. The DOR can be used as an effective performance indicator for the design of URLLC transmission schemes.

We assume that the transmitter has the full CSI to achieve the ideal continuous rate adaptive transmission. According to [15], the DOR is defined as the probability that the time required to successfully transmit a certain amount of data in a wireless channel is greater than a threshold duration $T_{\text {th }}$, which can be expressed as $\mathrm{DOR}=\operatorname{Pr}\left(\mathrm{DT}>T_{\mathrm{th}}\right)$, where $\mathrm{DT}=\frac{H}{B \cdot \log _{2}(1+\gamma)}$ represents the delivery time, $B$ is the channel bandwidth, and $H$ is the amount of data.

With (6), the DOR for the RIS-aided system can be readily evaluated as

$$
\operatorname{DOR}_{\mathrm{RIS}}=1-\mathrm{Q}_{\frac{1}{2}}\left(\frac{\sqrt{\lambda}}{\sigma}, \frac{\exp \left(\frac{H \cdot \ln (2)}{B \cdot T_{\text {th }}}\right)-1}{\sqrt{\bar{\gamma}} \sigma}\right) .
$$

Moreover, for the direct-link transmission system, $\mathrm{DOR}_{\mathrm{D}}$ can be exactly expressed as

$$
\operatorname{DOR}_{\mathrm{D}}=1-\mathrm{e}^{-\frac{\exp \left(\frac{H \cdot \ln (2)}{B \cdot T_{\mathrm{th}}}\right)-1}{\bar{\gamma}}}
$$

Finally, for the relaying system with the DF protocol, $\mathrm{DOR}_{\mathrm{R}}$ can be written as

$$
\operatorname{DOR}_{\mathrm{R}}=1-\mathrm{e}^{-\frac{\exp \left(\frac{H \cdot \ln (2)}{B \cdot \operatorname{th}_{h}}\right)-1}{2 \bar{\gamma}}} .
$$

\section{NUMERICAL RESULTS}

In this section, we present some numerical results to verify our analysis for the coverage distance, the PSG, and the DOR.

In Fig. 2, we plot the curves for the coverage distance of the RIS-aided systems, relaying systems, and direct link transmission when $N=4,6,8$ and $\gamma_{\text {th }}=2 \mathrm{~dB}$. We set $P_{\text {out }}=0.1$, which means that the reliability of the system link can maintain the required received SNR at least $90 \%$ of the time. In Fig. 2, we also plot the curve for the lower bound obtained from Eq. (8). From Fig. 2, we can see that the RIS-aided systems can obtain larger coverage distance in comparison with the relaying systems and the direct-link transmission. Also, increasing the value of $N$ can improve the system performance and expand coverage.

In Fig. 3, we plot the curves for the PSG. This figure shows the perfect match between the analysis and simulation

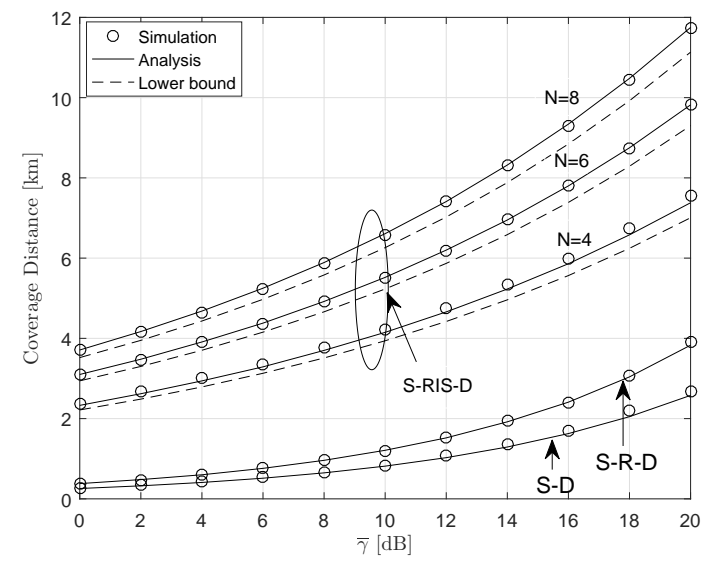

Fig. 2. Coverage distance comparison between RIS-aided systems, relaying systems, and direct link transmission.

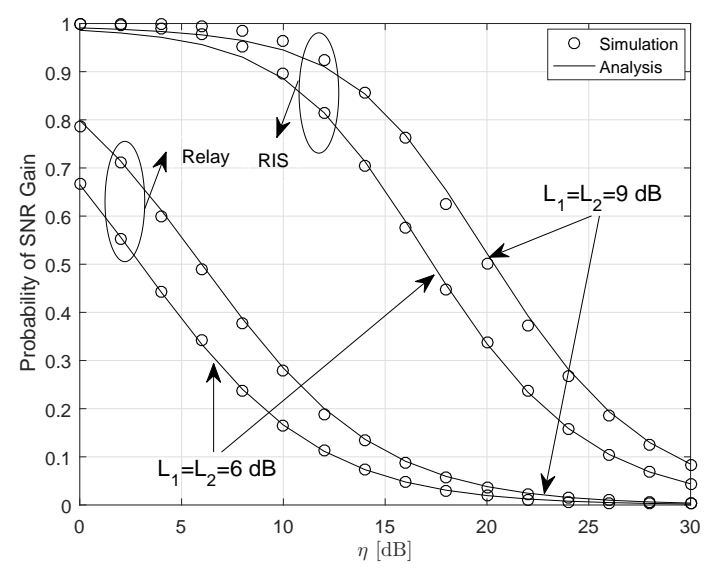

Fig. 3. Probability of SNR gain for relaying and RIS-aided systems when $L_{1}=L_{2}=6,9 \mathrm{~dB}$, and $N=4$.

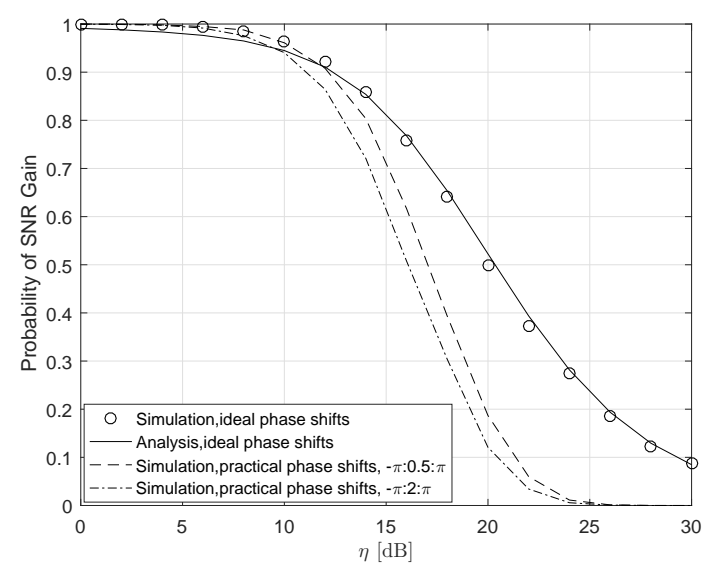

Fig. 4. Probability of SNR gain comparison for the RIS-aided systems with the ideal and practical phase shifts when $L_{2}=9 \mathrm{~dB}$, and $N=4$. 


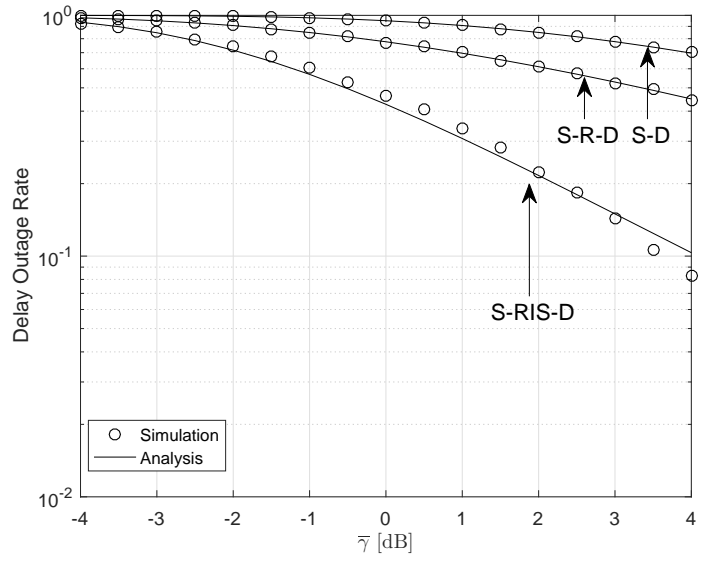

Fig. 5. DOR comparison between RIS-aided systems, relaying systems, and direct link transmission when $H=5 \mathrm{kbits}, B=2 \mathrm{MHz}$, and $T_{\mathrm{th}}=0.001 \mathrm{~s}$.

results. From Fig. 3, we can observe that the RIS-assisted system obtains a larger SNR gain than the relaying system, and it also shows that the SNR gain increases as the average SNR increases. For different RLGs, the higher RLGs have the greater PSG, which makes the performance of the system become better.

In Fig. 4, we present the PSG comparison between the ideal and practical phase shifts to observe the performance loss. From [9], $\varpi_{i}\left(\phi_{i}\right)=\left(1-\zeta_{\text {min }}\right)\left(\frac{\sin \left(\phi_{i}-\kappa\right)+1}{2}\right)^{\tau}+\zeta_{\text {min }}$, where $\zeta_{\min }$ is the minimum amplitude, $\kappa$ is the horizontal distance between $-\frac{\pi}{2}$ and $\zeta_{\min }$, and $\tau$ is the steepness of function curve. Without loss of generality, we set $\zeta_{\min }=0.8, \kappa=0.43 \pi$, and $\tau=1.6$. From Fig. 4, we can observe that there is a performance loss between the practical and ideal phase shifts. However, this performance loss can be improved by reducing the interval of discrete phase shifts.

Fig. 5 shows the DOR performance of the RIS-aided system, the relaying system, and the direct link transmission. From Fig. 5, it can be seen that the DOR performance of the three different transmission systems has been improved when increasing the average SNR. Moreover, using RISs can significantly improve the delay outage rate compared with other two transmission schemes.

\section{CONCLUSION}

In this paper, we analyzed the coverage, the PSG, and the DOR for the RIS-aided communication systems. The results revealed that the number of reflecting meta-surfaces has a great impact on the system performance, and that the use of RISs can provide a larger coverage, increase the SNR gain, and reduce the delay.

\section{REFERENCES}

[1] E. Basar, M. Di Renzo, J. De Rosny, M. Debbah, M. S. Alouini and R. Zhang, "Wireless communications through reconfigurable intelligent surfaces," IEEE Access, vol. 7, pp. 116753-116773, Aug. 2019.

[2] M. Di Renzo et al., "Smart radio environments empowered by reconfigurable AI meta-surfaces: An idea whose time has come," [Online]. Available: https://arxiv.org/abs/1903.08925.
[3] C. Liaskos, S. Nie, A. Tsioliaridou, A. Pitsillides, S. Ioannidis, and I. Akyildiz, "A new wireless communication paradigm through softwarecontrolled metasurfaces," IEEE Commun. Mag., vol. 56, no. 9, pp. 162169, Sep. 2018.

[4] Q. Wu and R. Zhang, "Towards smart and reconfigurable environment: Intelligent reflecting surface aided wireless network," IEEE Commun. Mag., vol. 58, no. 1, pp. 106-112, Jan. 2020.

[5] Q. Wu and R. Zhang, "Intelligent reflecting surface enhanced wireless network via joint active and passive beamforming," IEEE Trans. Wireless Commun., vol. 18, no. 11, pp. 5394-5409, Nov. 2019.

[6] Q. Wu and R. Zhang, "Beamforming optimization for wireless network aided by intelligent reflecting surface with discrete phase shifts," IEEE Trans. Wireless Commun., vol. 68, no. 3, pp. 1838-1851, Mar. 2020.

[7] C. Huang, A. Zappone, G. C. Alexandropoulos, M. Debbah and C. Yuen, "Reconfigurable intelligent surfaces for energy efficiency in wireless communication," IEEE Trans. Wireless Commun., vol. 18, no. 8, pp. 4157-4170, Aug. 2019.

[8] C. Huang, S. Hu, G. C. Alexandropoulos, A. Zappone, C. Yuen, R. Zhang, M. Di Renzo and M. Debbah, "Holographic MIMO surfaces for 6G wireless networks: Opportunities, Challenges, and Trends," [Online]. Available: https://arxiv.org/abs/1911.12296.

[9] S. Abeywickrama, R. Zhang, Q. Wu, C. Yuen, "Intelligent reflecting surface: practical phase shift model and beamforming optimization," [Online]. Available: https://arxiv.org/abs/2002.10112.

[10] L. Xiao, T. E. Fuja and D. J. Costello, "An analysis of mobile relaying for coverage extension," in Proc. IEEE ISIT, Aug. 2008, pp. 2262-2266.

[11] L. Yang, M. S. Alouini and K. Qaraqe, "On the performance of spectrum sharing systems with two-way relaying and multiuser diversity," IEEE Commun. Lett., vol. 16, no. 8, pp. 1240-1243, Aug. 2012.

[12] I. Lee and D. Kim, "Probability of SNR gain by dual-hop relaying over single-hop transmission in SISO Rayleigh fading channels," IEEE Commun. Lett., vol. 12, no. 10, pp. 734-736, Oct. 2008.

[13] I. Lee and D. Kim, "Probability of SNR gain by dual-hop MIMO relaying over single-hop Alamouti transmission," in Proc. IEEE ISWPMC, Nov. 2011, pp. 1-5.

[14] H. Yang and M. S. Alouini, "Data-oriented transmission in future wireless systems: Toward trustworthy support of advanced internet of things," IEEE Veh. Technol. Mag., vol. 14, no. 3, pp. 78-83, Sep. 2019.

[15] H. Yang, S. Choi and M. S. Alouini, "Ultra-reliable low-latency transmission of small data over fading channels: A data-oriented analysis," IEEE Commun. Lett., Dec. 2019.

[16] K. Ntontin, M. Di Renzo, J. Song, F. Lazarakis, J. D. Rosny, D. T. Phan Huy, O. Simeone, R. Zhang, M. Debbah, G. Lerosey, M. Fink, S. Tretyakov, S. Shamai, "Reconfigurable intelligent surfaces vs. relaying: Differences, similarities, and performance comparison," [Online]. Available: https://arxiv.org/abs/1908.08747.

[17] W. K. Tang, M. Z. Chen, X. Y. Chen, J. Y. Dai, Y. Han, M. Di Renzo, Y. Zeng, S. Jin, Q. Cheng, T. J. Cui, "Wireless communications with reconfigurable intelligent surface: Path loss modeling and experimental measurement," [Online]. Available: https://arxiv.org/abs/1911.05326.

[18] J. G. Proakis, M. Salehi, Digital Communications, 5th ed, New York: McGraw-Hill, 2008.

[19] V. M. Kapinas, S. K. Mihos, and G. K. Karagiannidis, "On the monotonicity of the meneralized Marcum and Nuttall Q-functions," IEEE Trans. Inform. Theo., vol. 55, no. 8, pp. 3701-3710, Aug. 2009.

[20] M. K. Simon and M. S. Alouini, "Exponential-type bounds on the generalized Marcum Q-function with application to error probability analysis over fading channels," IEEE Trans. Commun., vol. 48, no. 3, pp. 359-366, Mar. 2000.

[21] C. J. Chen and L. C. Wang, "Enhancing coverage and capacity for multiuser MIMO systems by utilizing scheduling," IEEE Trans. Wireless Commun., vol. 5, pp. 1148-1157, May 2006.

[22] G. Cui, L. Kong, X. Yang and D. Ran, "Two useful integrals involving generalised Marcum Q-function," Electronics Lett., vol. 48, no. 16, pp. 1017-1018, Aug. 2012.

[23] A. Nuttall, "Some integrals involving the $Q_{M}$ function (Corresp.)," IEEE Trans. Inform. Theo., vol. 21, no. 1, pp. 95-96, Jan. 1975. 